\title{
Nicotine replacement therapy and varenicline: A cohort study in low-income region (Monastir, Tunisia).
}

\author{
Asma Sriha Belguith ${ }^{* 1}$, Arwa Ben Salah ${ }^{1}$, Hanene Maatouk ${ }^{2}$, Ines Bouanene ${ }^{1}$, Hela Abroug ${ }^{1}$, Manel \\ Ben Fredj ${ }^{1}$, Mouna Safer ${ }^{1}$, Faycal Samaali ${ }^{3}$ and Mohamed Soltani ${ }^{1}$ \\ ${ }^{1}$ Community Medicine Department, University Hospital of Monastir, University of Medicine, Monastir, Tunisia \\ ${ }^{2}$ Family of Medicine Monastir, Tunisia \\ ${ }^{3}$ Head of Direction of Basic Health Care Tunis, National Tobacco Control, Tunisia
}

\begin{abstract}
Background: Among motivated Smokers, cessation can be performed by Nicotine Replacement Therapy (NRT), or by Varenicline (VAR). We aimed at determining quit rates among two cohorts, treated by NRT or VAR and, at identifying factors associated to quit and relapse delays.

Methods: We carried out a prospective cohort study at the smoking cessation center of the University Hospital of Monastir, in Tunisia from April 2009 to January 2012.

Results: 1042 active smokers have been followed in smoking cessation center between April 2009 and December 2010. One hundred eighty two participants had met the inclusion criteria and had agreed to participate in our study. Abstinence rates, were, $61.5 \%$ at 3 month follow up and $36.3 \%$ at one year $(\mathrm{p}=001)$. After adjusting on age, alcohol consumption, anxiety scale, and exhaled carbon monoxide values, there were no significant differences on abstinence rates in two groups NRT or VAR. In multivariate analysis, factors associated to a precipitate smoking relapse were presence of anterior previous attempts (HR: $0.10 ; 95 \%$ CI: 0.030-0.328), living with smokers at home (HR: $2.541 ; 95 \%$ CI: 1.019-6.336), having five following visits (HR: 0.091; 95\% CI: 0.017-0.500) and having minimal withdrawal syndrome reveled on follow up (HR: 0.23; 95\% CI: 0.08-0.662). Whereas, factors associated to quit delay was the use of NRT at the expense of VAR (HR: 4.966; 95\% CI: 2.81-8.76).

Conclusions: Relapse was not associated to treatment classes, whereas NRT have the benefit of a longer quit duration.
\end{abstract}

Keywords: Smoking cessation, Varenicline, Nicotine replacement therapy, Cohort study, Tunisia.

Abbreviations:

OR: Odds Ratio, CI95\%: Confidence Interval 95\%.

Accepted on 1 July, 2017

\section{Introduction}

Tobacco smoking is a global health problem. Worldwide, there are about 1 billion male and 250 million female smokers [1]. In Tunisia, half of men were daily tobacco smokers in 2013 [2], among them 37\% were Heavy Smokers HS (consumed 20 cigarettes per day and more) [3]. Most dependent smokers cannot successfully quit smoking because of nicotine addiction [4] that's why, despite of their lack of convincingness, they must need therapies [5]. Different smoking cessation therapies are now available such as Nicotine Replacement Therapy (NRT) and Varenicline (VAR). The NRT is an effective and a safe way to quit smoking. It has become the standard pharmacological treatment [6]. Likewise, VAR increase the chances of successful long-term smoking cessation between threefold compared with pharmacologically unassisted quit attempts, Hence it is a promising treatment option. [7-10]. Avoid the "Under-replacement" of standard doses of NRT among HS, by adjusting NRT dose depending on number of cigarettes consumed per day, is necessary to shun disappointing treatment efficacy results [11]. Studies on the effect of these two treatments on heavy smokers are rare. The objective of this study was to compare smoking cessation success and identify factors influencing quit and relapse delays.

\section{Methods}

\section{Study setting}

Fattouma Bourguiba University Hospital is a tertiary level teaching hospital. The center of smoking cessation, localized at the external consultation, is supporting by experienced specialists and provides quality services and treatment.

\section{Study area}

Monastir governorate is a tourism-oriented city, situated in the coastal region of Tunisia. It covers $0.7 \%$ area of all Tunisian territory and $5 \%$ of the Tunisian population live in. 


\section{Study cohort}

One thousand forty two active smokers, who presented to smoking cessation center between April 2009 and December 2010, have received pharmacological and behavioural therapy. Inclusion criteria were being HS (consuming more than 19 cigarettes per day); aged more than 18 years, motivated to quit and accepting to take part in this study including a follow-up examination every 2 weeks. Pregnant and breastfeeding women were not included.

\section{Method}

Inclusion runs over a period of nine months (April 2009December 2010). Period follow up, was over 24 weeks with a 2 weeks rhythm of visits. A phone survey was conducted on January 2012 to assess abstinence status. For phone interview, patients who attend follow up visits and used their medication regularly as prescribed, were paired on treatment NRT vs. VAR using electronic Random sampling (SPSS).

Nicotine replacement therapy: The aim of NRT is to replace nicotine from cigarettes, reducing withdrawal symptoms associated to smoking cessation and helping ex-smokers to resist the urge to smoke cigarettes [6]. In Tunisia available nicotine patch delivers nicotine through the skin in a steady dose. It' is available in 7, 14, and $21 \mathrm{mg}$ doses worn over 24 hours [12]. Hence, oral NRTs provide smokers with a coping strategy when cigarette cravings occur [7]. Combined NRT formulations have been shown to result in higher abstinence rates than single NRT. The effectiveness of NRT appears to be largely independent of the intensity of additional support provided to the smoker [6]. NRT can be used, in first step to reduce smoking, followed by complete quit and long-term abstinence [7]. Among HS, for avoiding the "Underreplacement" of standard doses of NRT, we have substituted one $\mathrm{mg}$ of nicotine provided by cigarettes consumed per day by one $\mathrm{mg}$ in NRT, than participant smoking 40 cigarettes per day was substituted by two nicotine patchs of $21 \mathrm{mg}$ associated to oral NRT [11].

Varinicline: Cytisine structure is behind the development of varenicline. His structure similar to nicotine and acetylcholine through their action as an agonist at $\alpha 4 \beta 2$ nAChRs, stimulates the release of dopamine [13]. The affinity of varenicline for the $\alpha 4 \beta 2$ nAChRs receptor is approximately three-fold and 16-fold greater than that of cytisine and nicotine. Consequently to its high affinity, it blocks nicotine-induced dopaminergic activation, potentially reducing the reward from smoking relapse. Smokers treated with varenicline should normally aim to quit approximately 1 week after the start of the treatment, a flexible quit-date is recommended [9].

Study interventions: At first visit, enrolled patients received a therapeutic education dealing with medication action and management. They were asked to choose between NRT and VAR. In the NRT group, the 24-h nicotine patch delivery system was used to aid smoking cessation. Subjects who smoked 40 cigarettes per day began with two $21 \mathrm{mg} /$ day nicotine patch, one per arm, for the first four weeks, two patch of $14 \mathrm{mg} /$ day in weeks five to eight, $21 \mathrm{mg} /$ day in weeks nine to twelve, and $14 \mathrm{mg} /$ day for four weeks finally $14 \mathrm{mg} /$ day until $20^{\text {th }}$ week. Nicotine tablet was used when needed to fight cravings. In the VAR group, patients received $0.5 \mathrm{mg} / \mathrm{d}$ VAR for three days, followed by $1 \mathrm{mg} / \mathrm{d}(0.5 \mathrm{mg}$ twice daily) for four days. On day 8 , the target quit date, the VAR dose was increased to $1 \mathrm{mg}$ twice daily until $12^{\text {th }}$ week.

Baseline assessments: We have collected demographic information (age, gender, educational level), smoking related information (daily number of cigarettes, previous attempts; other household smokers, alcohol use, level of smoking addiction (using the French version of the Fagerström test for nicotine dependence: FTND), Anxiety and depressive behaviour (using the French version of Hospital Anxiety and Depression Scale: HAD) [14]. We often have determined exhaled carbon monoxide levels.

Table 1. Participants' characteristics at baseline.

\begin{tabular}{|c|c|c|c|}
\hline & $\begin{array}{l}\text { VAR (91); n } \\
\text { (\%) }\end{array}$ & $\begin{array}{l}\text { NRT (91); n } \\
(\%)\end{array}$ & $\mathbf{p}$ \\
\hline Age: more than 40 years & $17(18.9)$ & $52(57.1)$ & 0 \\
\hline Gender: Male & $89(97.8)$ & $89(97.8)$ & 1 \\
\hline $\begin{array}{l}\text { Education: Bachelor degree and } \\
\text { higher }\end{array}$ & $48(54.5)$ & $41(45.1)$ & 0.2 \\
\hline Previous attempt & $69(76.7)$ & $67(73.6)$ & 0.64 \\
\hline Live with home smokers & $33(38.8)$ & $28(30.8)$ & 0.26 \\
\hline Alcohol use & $27(30.3)$ & $2(09.5)$ & 0.05 \\
\hline Fagerström scale: 7 and more & $57(62.6)$ & $68(74.7)$ & 0.08 \\
\hline Anxiety scale: More than 11 & $19(22.6)$ & $9(09.9)$ & 0.02 \\
\hline Depression scale: More than 11 & $3(03.6)$ & $0(00.0)$ & 0.07 \\
\hline \multicolumn{4}{|l|}{ Cigarettes consumed per day (\%). } \\
\hline $20-30$ & $64(70.3)$ & $58(63.7)$ & 0.34 \\
\hline 31 and more & $27(29.7)$ & $33(36.3)$ & \\
\hline One co-morbidity and more & $17(18.7)$ & $21(23.1)$ & 0.47 \\
\hline Have a morning cough & $32(35.2)$ & $24(26.4)$ & 0.2 \\
\hline Have an effort dyspnea & $44(48.4)$ & $45(49.5)$ & 0.94 \\
\hline ECM; median, IIQ (ppm) & $15(7.0)$ & $18(13.0)$ & 0 \\
\hline
\end{tabular}

VAR: Varenicline; NRT: Nicotine Replacement Therapy; ECM: Exhaled Carbon Monoxide; ppm: Particles Per Million

Assessment: Smoking status and adverse effects were notified at follow up visits and at the phone survey. Smoking status was assessed by self-report of smoking in the preceding 7 days. Self-reported abstinence was confirmed using biochemical validation via exhaled carbon monoxide levels (cut off: $8 \mathrm{ppm}$ ) [3]. The adverse effects included those related to quit (urges to smoke, headaches, feeling nervous, memory impairment, sleep disorder, increased appetite) and those related to the treatment (gastric ache, nausea and dermal reaction) [4]. 
Citation: Belguith AS, Salah AB, Maatouk H, et al. Nicotine replacement therapy and varenicline: A cohort study in low-income region (Monastir, Tunisia). Allied J Med Res 2017;1(1):16-22.

\section{Statistical analyses}

Analyses performed with SPSS for Windows version 20.0. Data was expressed in terms of proportions, for categorical variables, means and standard deviation for quantitative variables. Categorical variables were compared with Pearson $\chi^{2}$-test. We have performed an analysis adjustment for confounders using logistic regression. We have used a multivariate survival analyses (Cox regression) to identify predictors of relapses and of quit. In fact in our longitudinal study smokers could make quit attempts at different times during the follow-up, and they also may have different lengths of follow-up. This method allows for differential follow-up and efficiently accommodates missing data due to censoring. First we have performed Kaplan-Meier method including all factors associated with the outcome, second factors were included in the Cox regression model when $\mathrm{p} \leq 0.20$. Findings were considered statistically significant at $\mathrm{p}<0.05$.

Table 2. Quit rates and adverse effects among study participants.

\begin{tabular}{|c|c|c|c|}
\hline & Varenicline & NRT & $\mathbf{P}^{*}$ \\
\hline \multicolumn{4}{|c|}{ Smoking cessation at follow up } \\
\hline No: $n(\%)$ & $46(50.5)$ & $24(26.4)$ & $0.251^{*}$ \\
\hline YES: n (\%) & $45(49.5)$ & $67(73.6)$ & \\
\hline \multicolumn{4}{|c|}{ Smoking cessation at one year } \\
\hline No: $n(\%)$ & $67(73.6)$ & $49(53.8)$ & $0.646^{*}$ \\
\hline YES: n (\%) & $24(26.4)$ & $42(46.2)$ & \\
\hline \multicolumn{4}{|l|}{ Adverse effects } \\
\hline No: n (\%) & $46(50.5)$ & $23(25.3)$ & \\
\hline One & $23(30.3)$ & $30(33.0)$ & \\
\hline More than one & $22(28.9)$ & $38(41.7)$ & \\
\hline \multicolumn{4}{|c|}{ Adverse effects related to quit } \\
\hline Urges to smoke & $4(05.5)$ & $23(25.3)$ & \\
\hline Headaches & $6(08.3)$ & $17(18.7)$ & \\
\hline Feeling nervous & $19(26.4)$ & $2(02.2)$ & \\
\hline Memory impairment & $7(09.7)$ & $1(01.1)$ & \\
\hline Sleep disorder & $6(08.3)$ & $13(14.3)$ & \\
\hline Increased appetite & $4(05.5)$ & $5(05.5)$ & \\
\hline \multicolumn{4}{|c|}{ Adverse effects related to treatment } \\
\hline Gastric ache & $24(33.3)$ & $1(01.1)$ & \\
\hline Dermal Reaction & 0 & $11(12.1)$ & \\
\hline
\end{tabular}

*: Adjusted on age, Alcohol use, Anxiety scale, Exhaled carbon monoxide values

Table 3. Factors associated to relapses delay in a cohort of smokers consuming 20 cigarettes and more in a low income region (Monastir; Tunisia).

HR CI 95\% HR p

\begin{tabular}{|c|c|c|c|c|}
\hline \multicolumn{5}{|c|}{ Treatment classes } \\
\hline \multirow{2}{*}{$\begin{array}{l}\text { VAR } \\
\text { NRT }\end{array}$} & \multicolumn{4}{|l|}{1} \\
\hline & 0.701 & 0.392 & 1.25 & 0.231 \\
\hline \multicolumn{5}{|c|}{ Anterior previous attempts } \\
\hline \multicolumn{5}{|l|}{ No } \\
\hline Yes & 0.1 & 0.03 & 0.33 & 0 \\
\hline \multicolumn{5}{|c|}{ Living with smokers at home } \\
\hline No & 1 & & & \\
\hline Yes & 2.541 & 1.019 & 6.34 & 0.046 \\
\hline \multicolumn{3}{|c|}{ number of follow up visits } & & 0.013 \\
\hline 2 & 0.214 & 0.053 & 0.86 & 0.03 \\
\hline 3 & 1.38 & 0.433 & 4.4 & 0.585 \\
\hline 4 & 0.198 & 0.034 & 1.14 & 0.069 \\
\hline 5 & 0.091 & 0.017 & 0.5 & 0.006 \\
\hline \multicolumn{4}{|c|}{ Number of difficulties during smoking cessation } & 0.037 \\
\hline 1 & 0.23 & 0.08 & 0.66 & 0.006 \\
\hline 2 & 0 & 0 & & 0.98 \\
\hline 3 & 0.23 & 0.046 & 1.15 & 0.073 \\
\hline
\end{tabular}

Variables included in multivariate model were: Age of first cigarette; Anterior previous attempts, Living with smokers at home, Importance of smoking cessation, self confidence in quitting smoking, treatment classes (NRT/ VAR), number of follow up visits, Number of difficulties during smoking cessation, level of study (baccalaureate).

Table 4. Factors associated to quit delay in a cohort of smokers consuming 20 cigarettes and more in a low income region (Monastir; Tunisia).

\begin{tabular}{lllll}
\hline \multicolumn{1}{l}{ HR } & Cl 95\% & HR & p \\
\hline Treatment classes & & & & \\
\hline VAR & 1 & & & \\
\hline NRT & 4.966 & 2.814 & 8,764 & 0,000 \\
\hline Self-importance of smoking cessation (scale $0-10)$ & & & \\
\hline$<8$ & 1 & & & \\
\hline$\geq 8$ & 1150 & 0,955 & 1,384 & 0,140 \\
\hline Being abstinent & 1 & & & \\
\hline Less than 4th follow up visit & 0,906 & 0,565 & 1,452 & 0,682 \\
\hline 4th follow up visit and more & & & & \\
\hline
\end{tabular}

Variables included in multivariate model were: treatment classes (NRT/VAR), Self-importance of smoking cessation, Being abstinent at 4th follow up visit, gain follow up, disponibilité ttt, comorbidité

\section{Results}

From April 2009 to December 2010, a total of 1042 HS were assessed. One hundred eighty two were eligible to study criteria. Four women were included $(2.2 \%)$. Mean age was 37.8 years (SD: 11.8$)$. Out of these $91(50 \%)$ received VAR. 
Table 1 shows smokers characteristics at baseline, patients in VAR cohort was younger and more anxious than those treated with NRT. Abstinence rates, were, $61.5 \%$ at the latest followup visit and $36.3 \%$ at one year $(\mathrm{p}=0.01)$. Smoking cessation rates, adjusted by age, alcohol consumption, anxiety, and ECM values, at one year were statistically comparable between the two groups NRT or VAR (The quit rates were $46.2 \%$ and $26.4 \%$ respectively with NRT and VAR at one year $(\mathrm{p}=0.646$; adjusted OR 1.28; CI 95\%: 0.44-3.74).

Likewise adverse effects rates were similar in two groups. Urge to smoke and feeling nervous were the most adverse effects, related to quit, described respectively in NRT $(25.3 \%)$ and VAR cohorts (26.4\%) (Table 2). In multivariate analysis, factors associated to smoking relapse at one year were presence of anterior previous attempts (HR: $0.10 ; 95 \% \mathrm{CI}$ : 0.030-0.328), living with smokers at home (HR: $2.541 ; 95 \%$ CI: 1.019-6.336), having five following visits (HR: 0.091; 95\% CI: 0.017-0.500) and having minimal withdrawal syndrome revealed on follow up (HR: 0.23; 95\% CI: 0.08-0.662) (Table 3 ). Factors associated to quit delay were the use of NRT at the expense of VAR (HR: 4.97; 95\% CI: 2.81-8.76) (Table 4).

Table 5. Rates of success in smoking cessation in literature.

\begin{tabular}{|c|c|c|c|c|c|c|}
\hline Authors & Comparison & $\mathbf{n}$ & Survey date & VAR (\%) & NRT (\%) & OR $(95 \% \mathrm{Cl})$ \\
\hline Gray KM (20) & VAR/NRT & $67 / 73$ & 4 weeks & 9.6 & 22.4 & \\
\hline Cahill K (21) & VAR/NRT & & 26 weeks & 27.6 & 31.5 & $1.57(1.29-1.91)$ \\
\hline Kaduri, P (16) & VAR/NRT & $98 / 98$ & & 33.7 & 18.4 & $1.71 ; 1.05-2.79$ \\
\hline Koegelenberg CF (17) & NRT+VAR/VAR & 435 & 24 weeks & 32.6 & $49.0^{* *}$ & $1.98 ; 1.25-3.14$ \\
\hline Kotz D (22) & VAR/NRT & $118 / 152$ & 24 weeks & 39.8 & 19.7 & $3.83(1.88-7.77) £$ \\
\hline Hsueh (23) & VAR/NRT & & 144 weeks & 18.9 & 7.6 & $7.94(1.87-33.74) £$ \\
\hline Our study (\%) & NRT/VAR & $91 / 91$ & 52 week & 26 & 46 & $1.28(0.44-3.74)$ \\
\hline Aubin $\mathrm{HJ}(9)$ & VAR/ NRT & $376 / 370$ & 24-52 week & 26.1 & 20.3 & $1.40(0.99-1.99) £$ \\
\hline
\end{tabular}

*: Varenicline or Bupropion, ${ }^{* \star} \mathrm{NRT}+$ Varenicline, $£$ Varenicline versus NRT;

\section{Discussion}

We have determined that the overall success rate was $36.3 \%$ at one year and that adjusted abstinence rates were similar in two groups NRT vs. VAR. Likewise we have identified associated factors to quit and to relapse among participants consuming 20 cig per day and more.

Our finding highlights that younger, drinkers and anxious HS smokers have significantly chosen VAR at the expense of NRT, similarly Aubin H J, in his review, has described that VAR was preferred to NRT [9]. The quit rates were $46.2 \%$ and $26.4 \%$ respectively with NRT and VAR (adjusted $\mathrm{p}=0.646$ ). Our results were similar to those described by Aubin HJ [9]. Gray $\mathrm{KM}$ and Cahill $\mathrm{K}$ have described the superiority of NRT versus VAR [14-16]. Kaduri P, Kotz D and Hsueh KC, have revealed higher efficacy of VAR (Table 5) [17-19]. Those differences in results can be explained by ethnic [20], gender [21,22], degree of dependence [3] and the study design. Koegelenberg D and Chang $\mathrm{PH}$ have established advantageous rates by the association of NRT and VAR [22,23]. Also, Ramon JM had demonstrated better results of abstinence at $24^{\text {th }}$ week with combination NRT and VAR among smokers consuming more than 29 cig per day (OR 1.46; 95\% CI 1.2-2.8) [24]. The abstinence rates at one year, among our cohorts, were equivalent to that described by Kapaya with NRT [25].

In our study, urge to smoke was the major withdrawal symptom perceived with NRT. The combination of dermal and oral NRT may reduce this disadvantage [26]. Aubin has illustrated that NRT safety and tolerability is probably the best among the two first line medications for smoking cessation [9]. Urge to smoke was rarely perceived by the VAR cohort, may be, because of its ability to partially substitute the reinforcement-enhancing effects of nicotine [27]. Therefore, VAR had reduced significantly the withdrawal symptoms and craving compared to NRT [28]. Among our VAR cohort, feeling nervous was the most dis-advantage perceived and we haven't registered serious side effects. The most frequent adverse events, described in the literature with VAR, were nausea [17,28], abnormal dreams and headaches [23]. Hartmann-Boyce had notified that there's no evidence of a significant increase in serious adverse events in trial participants randomized to VAR when compared to placebo controls [29]. The abstinent rates were higher at follow up than at one year with two treatments. By stopping monitoring, exsmokers risked relapse, were left in environment encouraging tobacco consumption [2]. That's why; we propose managing tobacco consumption as a chronic disease, extending a standardized follow up for cessation until one year. Fiore $\mathrm{MC}$, had recommended, a smoking reduction with NRT for few months, in the perspective to prepare smokers to make an attempt, with best chance of success [30]. In our study, we adjusted the patch's doses in terms of the number of cigarettes smoked, by substituting each cigarette by one $\mathrm{mg}$ in the transdermal nicotine patch. Thereby, we substitute 40 cigarettes per day by two patches of $21 \mathrm{mg}$ per $24 \mathrm{~h}$. With VAR, for smokers of 40 cigarettes per day, maybe some nicotine receptors are not saturated and will not disaccustom. This "under-dosing" could 
Citation: Belguith AS, Salah AB, Maatouk H, et al. Nicotine replacement therapy and varenicline: A cohort study in low-income region (Monastir, Tunisia). Allied J Med Res 2017;1(1):16-22.

be the cause of less abstinence rates among participants, high smokers, treated by VAR.

Several studies were interested to abstinence related factors and relapse. In our longitudinal study, factors decreasing relapse risk were presence of anterior previous attempts, having five following visits and minimal withdrawal syndrome reveled on follow up, while living with smokers at home increase this risk. Factors associated to quit at one year was the use of NRT at the expense of VAR. Chaiton $M$ has demonstrated that exposure to smoking on patios of a bar or restaurant is associated with a high risk of relapse [31]. Halonen has indicated that living near a tobacco shop increases this risk [32]. Brennan has showed the interest of anti-tobacco television advertisements, on behavior change [33]. Narwani has indicated that the absence of co-morbidity, raise the likelihood to improve the period of abstinence, when treatment is finished [34]. Schwartz has criticized the important health management among sick smokers [35] we suggest, as Wilby that treatment should not be routinely prescribed to ill patients even in poor health [36] because implication of smokers in cessation process is very needful [37]. Authors recommended taking into consideration a refund or free treatment $[38,39]$. Cropsey et al. have indicated that providing medication increase cessation rates in criminal justice individuals supervised in the community [40]. Our results describing predictor factors associated to quit and to relapse in Tunisia, which is a low income country, were analogous to those described by Courtney RJ among low-socioeconomic status smokers in Australia [41]. Several studies must be conducted to explore the newly outbreak of electronic cigarettes [43].

Our study has many strengths and limitations that warrant discussion. Using a data cohort study over two years study period has allowed us the use of robust statistical methods to characterize the factors influencing abstinence in VAR and NRT cohort and those associate with the longer period of quit. Our analysis enabled us to suggest an available treatment in Tunisia among GS. However we are not able to assess gender effect that may influence the choice and rates of abstinence because of the lack of women smokers especially, those consuming more than 20 cigarettes per day in the study region.

\section{Conclusion}

High dependent smokers can be treated each with NRT and VAR. Number of adverse effects was less with VAR. NRT increase quit delay. We highlighted that we could managed tobacco consumption as a chronic disease, extending a standardized follow up during a period of one year and more.

\section{Ethics Approval and Consent to Participate}

We had an oral approval for smokers to participate in this study. We had not found refusal cases to take part in the study.

\section{Acknowledgements}

For the entire team of preventive medicine and epidemiology, as well as interns and residents, who, participated in data collection and consultation management. The Tunis direction of basic health care for the supply of medication, specially Dr Nabli Mounira for his availability and kindness. We offer this paper to our eminent Professor Soltani Mohammed, and the department head, who died in March 2016.

\section{Funding}

We have not used a funding for the research. The design of the study and collection, analysis, and interpretation of data and the writing of the manuscript was conducted by doctors in the smoking cessation center during their hours of work (public system with a lump payment). Treatments were well-stocked by Tunis direction of basic health care, managed by Nabli Mounira in 2011.

\section{References}

1. Lando HA, Hipple BJ, Muramoto M, et al. Tobacco is a global paediatric concern. Bull World Health Organ. 2010;88:2.

2. WHO Report on the Global Tobacco Epidemic, 2013 Country profile Tunisia.

3. Sriha BA, Bouanene I, Elmhamdi S, et al. La dépendance nicotinique et l'intoxication au monoxyde de carbone chez les fumeurs adultes. Tun Méd. 2015;4:231-6.

4. Xiao D, Chu S, Wang C. Smoking cessation in Asians: focus on varenicline. Patient Prefer Adherence. 2015;9:579-84.

5. Hollands GJ, McDermott MS, Lindson-Hawley N, et al. Interventions to increase adherence to medications for tobacco dependence. Cochrane Database Syst Rev. 2015;009164.

6. Silagy C, Lancaster T, Stead L, et al. Nicotine replacement therapy for smoking cessation. Cochrane Database Syst Rev. 2004;000146.

7. Cahill K, Stead LF, Lancaster T. Nicotine receptor partial agonists for smoking cessation. Cochrane Database Syst Rev. 2008;3:006103.

8. Sriha BA, Elmhamdi S, Buanene I, et al. Les facteurs prédictifs de rechute tabagique chez les adultes bénéficiaires d'aide au sevrage. Tun Méd. 2015;1:21-7.

9. Aubin HJ, Luquiens A, Berlin I. Pharmacotherapy for smoking cessation: pharmacological principles and clinical practice. Br J Clin Pharmacol. 2014; 77:324-36.

10. Kumar R, Prasad R. Smoking cessation: an update. Indian J Chest Dis Allied Sci. 2014;56:161-9.

11. Ebbert JO, Post JA, Moyer TP, et al. Nicotine Percentage Replacement Among Smokeless Tobacco Users with Nicotine Patch. Drug Alcohol Depend. 2007;89:223-6.

12. Bailey SR, Crew EE, Riske EC, et al. Efficacy and Tolerability of Pharmacotherapies to Aid Smoking Cessation in Adolescents. Paediatr Drugs. 2012;14:91-108.

13. Williams JM, Steinberg MB, Steinberg ML, et al. Varenicline for tobacco dependence: panacea or plight? Expert Opin Pharmacother. 2011;12:1799-812. 
14. Elmhamdi S, Sriha BA, Bouanene I, et al. Predictors of smoking relapse in a cohort of adolescents and young adults in Monastir (Tunisia). Tob Induc Dis. 2013;11:12.

15. Gray KM, McClure EA, Baker NL, et al. An exploratory short-term double-blind randomized trial of varenicline versus nicotine patch for smoking cessation in women. Addiction. 2015;110:1027-34.

16. Cahill K, Stevens S, Lancaster T. Pharmacological treatments for smoking cessation. JAMA. 2014; 8;311:193-4.

17. Kaduri P, Voci S, Zawertailo L, et al. Real-world effectiveness of varenicline versus nicotine replacement therapy in patients with and without psychiatric disorders. J Addict Med. 2015;9:169-76.

18. Kotz D, Brown J, West R. Prospective cohort study of the effectiveness of varenicline versus nicotine replacement therapy for smoking cessation in the "real world". BMC Public Health. 2014;14:1163.

19. Hsueh KC, Hsueh SC, Chou MY, et al. Varenicline versus transdermal nicotine patch: a 3-year follow-up in a smoking cessation clinic in Taiwan. Psychopharmacology. 2014;231:2819-23.

20. Hooper MW, Baker EA, de Ybarra DR, et al. Acculturation predicts 7-day smoking cessation among treatment-seeking African-Americans in a group intervention. Ann Behav Med. 2012;43:74-83.

21. Berlin I, Grange G, Jacob N, et al. Nicotine patches in pregnant smokers: randomised, placebo controlled, multicentre trial of efficacy. BMJ. 2014;348:1622.

22. Chang $\mathrm{PH}$, Chiang $\mathrm{CH}$, Ho $\mathrm{WC}$, et al. Combination therapy of varenicline with nicotine replacement therapy is better than varenicline alone: a systematic review and meta-analysis of randomized controlled trials. BMC Public Health. 2015;15:689.

23. Koegelenberg CF, Noor F, Bateman ED, et al. Efficacy of varenicline combined with nicotine replacement therapy vs varenicline alone for smoking cessation: a randomized clinical trial. JAMA. 2014;312:155-61.

24. Ramon JM, Morchon S, Baena A, et al. Combining varenicline and nicotine patches: a randomized controlled trial study in smoking cessation. BMC Med. 2014;12:172.

25. Kapaya M, Tong V, Ding H. Nicotine replacement therapy and other interventions for pregnant smokers: Pregnancy Risk Assessment Monitoring System, 2009-2010. Prev Med. 2015;78:92-100.

26. Jiloha RC. Pharmacotherapy of smoking cessation. Indian J Psychiatry. 2014;56:87-95.

27. Levin ME, Weaver MT, Palmatier MI, et al. Varenicline dose dependently enhances responding for non pharmacological reinforces and attenuates the reinforcement-enhancing effects of nicotine. Nicotine Tob Res. 2012;14:299-305.

28. Ashare RL, Tang KZ, Mesaros AC, et al. Effects of 21 days of varenicline versus placebo on smoking behaviors and urges among non-treatment seeking smokers. J Psychopharmacol. 2012;26:1383-90.
29. Hartmann-Boyce J, Stead LF, Cahill K, et al. Efficacy of interventions to combat tobacco addiction: Cochrane update of 2013 reviews. Addiction. 2014;109:1414-25.

30. Fiore MC, Baker BT. Treating smokers in the health care setting. N Engl J Med. 2011;365:1222-31.

31. Chaiton M, Diemert L, Zhang B, et al. Exposure to smoking on patios and quitting: a population representative longitudinal cohort study. Tob Control. $2014 ; 28$.

32. Halonen JI, Kivimaki M, Kouvonen A, et al. Proximity to a tobacco store and smoking cessation: a cohort study. Tob Control. 2014;23:146-51.

33. Brennan E, Durkin SJ, Wakefield MA, et al. Assessing the effectiveness of antismoking television advertisements: do audience ratings of perceived effectiveness predict changes in quitting intentions and smoking behaviours? Tob Control. 2014;23:412-8.

34. Narwani V, Harries M. Treatment modality: a predictor of continued tobacco use after treatment in patients with laryngeal cancer. J Laryngol Otol. 2014;128:153-8.

35. Schwartz MD, Jensen A, Wang B, et al. Panel Management to Improve Smoking and Hypertension Outcomes by VA Primary Care Teams: A ClusterRandomized Controlled Trial. J Gen Intern Med. 2015;30:916-23.

36. Wilby KJ, Harder CK. Nicotine replacement therapy in the intensive care unit: a systematic review. J Intensive Care Med. 2014;29:22-30.

37. De Viron S, Malats N, Van der HJ, et al. Environmental and genomic factors as well as interventions influencing smoking cessation: a systematic review of reviews and a proposed working model. Public Health Genomics. 2013;16:159-73.

38. Canas A, Alba LH, Becerra N, et al. Efficacy and safety of medication use for the cessation of tobacco addiction: A review of Clinical Practice Guidelines. Rev Salud Publica. 2014;16:772-85.

39. Raupach T, van Schayck CP. Pharmacotherapy for smoking cessation: current advances and research topics. CNS Drugs. 2011;25:371-82.

40. Cropsey KL, Clark CB, Stevens EN, et al. Predictors of medication adherence and smoking cessation among smokers under community corrections supervision. Addict Behav. 2017;65:111-7.

41. Courtney RJ, Clare P, Boland V, et al. Predictors of retention in a randomised trial of smoking cessation in low-socioeconomic status Australian smokers. Addict Behav. 2017;64:13-20.

42. Bullen $\mathrm{CH}$, Williman J, Howe C, et al. Study protocol for a randomised controlled trial of electronic cigarettes versus nicotine patch for smoking cessation. BMC Public Health. 2013;13:8.

\section{*Correspondence to:}

Asma Sriha Belguith

Associate Professor 
Citation: Belguith AS, Salah AB, Maatouk H, et al. Nicotine replacement therapy and varenicline: A cohort study in low-income region (Monastir, Tunisia). Allied J Med Res 2017;1(1):16-22.

Preventive Medicine and Epidemiology Service,

Community Medicine Department,

University of Medicine, Monastir,
Tel: 21697238022 ,

E-mail: belguith_asma@yahoo.fr 